\title{
CARACTERÍSTICAS MORFOMÉTRICAS DE ÓRGANOS LINFOIDES Y ESTUDIOS SEROLÓGICOS EN LEVANTE DE PONEDORAS UTILIZANDO UN INMUNOMODULADOR, VITAMINAS Y AMINOÁCIDOS
}

\author{
Luz S. Cortés Machado*, MVZ, Esp. ., Sonia C. Villamarín Jurado, MVZ
}

${ }_{1}$ Facultad de Medicina Veterinaria y Zootecnia, Universidad Cooperativa de Colombia, Colombia

Recibido: 11 de marzo del 2013 Aprobado: 10 de mayo del 2013

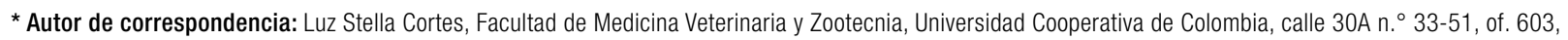
(57) 7 6356624, Bucaramanga, Colombia, correo electrónico: luz.cortes@ucc.edu.co

Cómo citar este artículo: Cortés-Machado LS, Villamarín-Jurado SC. Características morfométricas de órganos linfoides y estudios serológicos en levante de ponedoras utilizando un inmunomodulador, vitaminas y aminoácidos. Spei Domus. 2013; 9(18): 29-36.

Resumen. El primer órgano blanco que lesiona el virus de la enfermedad infecciosa de la bolsa (IBDv) es la bolsa de Fabricio, que manifiesta una depleción severa de las células B. Fundamentados en la relación existente entre la morfometría de los órganos linfoides y la inmunocompetencia de las aves, es de interés observar la acción que pueden ejercer los inmunomoduladores, vitaminas y aminoácidos en la respuesta inmune para la enfermedad de Gumboro (IBD). En los órganos linfoides (timo, bazo y bolsa de Fabricio) se evaluaron hasta las primeras ocho semanas de edad los siguientes parámetros: peso del ave, peso de cada órgano y serologías para la entidad de Gumboro a cinco y nueve semanas. Se dividieron en dos grupos con el mismo manejo (alojamiento, alimentación y ambiente), pero con tratamientos diferentes. El grupo A (prueba J8), con 55.062 aves, recibió inmunomodulador los tres primeros días de vida y los dos días posteriores a cada una de las aplicaciones de la vacuna de Gumboro. Las vitaminas y aminoácidos se administraron del cuarto al sexto día de vida y dos días posteriores al cambio de alimento (fase de preiniciación e iniciación). Al grupo B (control J7), con 55.484 aves, se administró protector hepático; para los dos grupos se tomaron muestras serológicas a cinco y nueve semanas de edad. En conclusión, el uso del inmunomodulador, vitaminas y aminoácidos durante el protocolo de recepción presenta beneficios que se pueden observar en el mejor consumo, peso, uniformidad y buen desarrollo del sistema inmune.

Palabras clave: Gumboro, inmunocompetencia, órganos linfoides, serología.

\section{Morphometric Characteristics of Lymphoid Organs and Serological Studies in Egg-Laying Birds Using an Immunomodulator, Vitamins and Amino Acids}

\begin{abstract}
The first target organ damaged by infectious bursa virus disease (IBVD) is the bursa, manifested in severe depletion of the B cells. However, this depletion depends on the regions of the lymphoid organs involved in the infection. Based on the existing relationship between the morphometry of the lymphoid organs and the inmunocompetence of the birds, it is interesting to observe possible effects from immunomodulators, vitamins and amino acids on the immune response for Gumboro (IBD) disease. In the lymphoid organs (swindle, spleen and bursa), the following parameters were evaluated until the first 8 weeks of age: weight of the bird, weight of each organ and serology for the Gumboro entity at 5 and 9 weeks. They were divided into 2 groups with the same handling (accommodation, feed and environment), but with different treatments. Group A (test J8), consisting of 55,062 birds, was given an immunomodulators during the first 3 days of life and 2 days after each application of the Gumboro vaccine. The vitamins and amino acids were administered from the fourth to the sixth day and 2 days following the change in food (pre-initiation and initiation phases). Group B (Control j7), consisting of 55,484 birds, was administered liver protector. Serological samples were taken at 5 and 9 weeks of age. In conclusion, the use of the immunomodulator, vitamins and amino acids during the reception protocol produces benefits observed in better consumption, weight, uniformity and proper development of the immune system.
\end{abstract}

Keywords: Gumboro, inmunocompetence, lymphoid organs, serology.
Características morfométricas de órgãos linfoides e estudos serológicos em levantamento de poedeiras utilizando um imunomodulador, vitaminas e aminoácidos

Resumo. O primeiro órgão branco que lesiona o vírus da doença infecciosa da bolsa (IBDV) é a bursa de Fabrício, que manifesta uma depleção severa das células B. Fundamentados na relação existente entre a morfometria dos órgãos linfoides e a imunocompetência das aves, é de interesse observar a ação que podem exercer os imunomoduladores, vitaminas e aminoácidos na resposta imune para a doença de Gumboro (IBD). Nos órgãos linfoides (timo, baço e bursa de Fabrício), avaliaram-se até as primeiras oito semanas de idade os seguintes parâmetros: peso da ave, peso de cada órgão e serologias para a entidade de Gumboro a cinco e nove semanas. Dividiram-se nos dois grupos com o mesmo manejo (alojamento, alimentação e ambiente), mas com tratamentos diferentes. O grupo A (prova J8), com 55.062 aves, recebeu imunomodulador nos três primeiros dias de vida e nos dois dias posteriores a cada uma das aplicações da vacina de Gumboro. As vitaminas e aminoácidos se administraram do quarto ao sexto dia de vida e dois dias posteriores à mudança de alimento (fase de pré-iniciação e iniciação). Ao grupo B (controle J7), com 55.484 aves, administrou-se protetor hepático; para os dois grupos se tomaram amostras serológicas a cinco e nove semanas de idade. Em conclusão, o uso do imunomodulador, vitaminas e aminoácidos durante o protocolo de recepção apresenta benefícios que se podem observar no melhor consumo, peso, uniformidade e bom desenvolvimento do sistema imune.

Palavras-chave: Gumboro, imunocompetência, órgãos linfoides, serologia. 


\section{Introducción}

En las últimas dos o tres décadas el sector avícola ha crecido drásticamente y sigue creciendo para satisfacer la alta demanda que existe para este tipo de proteína. Sin embargo, no se puede crecer exitosamente si no se mantiene un buen estado de salud [1]. En este estudio se pretendió evaluar las características morfométricas de los órganos linfoides, evaluados en las primeras semanas de vida del ave, especialmente frente a la enfermedad de Gumboro, por ser una enfermedad inmunodepresora.

Las aves se encuentran diariamente enfrentadas a retos de agentes patógenos presentes en las granjas avícolas que permiten la presencia de problemas de tipo inmunosupresivos cuando estas se encuentran desafiadas y su sistema inmune no responde de manera efectiva. Por esto surge la necesidad de buscar diferentes técnicas que permitan tener con más detalle el estatus sanitario de nuestra parvada. En el estudio realizado en el estado de Zulia, Venezuela, con pollos de engorde de la línea Ross, se llevó a cabo una evaluación morfométrica (timo, bazo y bursa) y de diámetro (bursómetro) que permitió definir un alto coeficiente de correlación entre cada una de las variables y el peso del ave, junto con el tamaño de sus órganos linfoides [2]. Por otra parte, este estudio permite definir un parámetro de referencia sobre los índices morfométricos en la zona de Lebrija, Santander, y puede ser una herramienta económica que permita la toma de decisiones respecto al estado inmunocompetente de las aves.

La depleción linfoide es un síndrome clínico que se caracteriza en las aves por una disminución o ausencia de células linfoides, y es conocido también como inmunosupresión. Existen diferentes factores relacionados con la presencia de esta condición, que pueden ser de tipo infeccioso o no infeccioso. En el primero están los medicamentos, la temperatura ambiental y el estrés; por otra parte, dentro del segundo grupo, se encuentran micotoxinas inmunodepresoras, agentes virales (enfermedad de Marek, Gumboro, Reovirus) y toxinas bacterianas. La capacidad de un organismo para sintetizar y producir anticuerpos puede verse afectada por una disfunción temporal o permanente de su sistema inmune. Los cambios que se generan en diversas sustancias del sistema de defensa del ave como citosinas, interferón y proteínas del complemento disminuyen la capacidad quimiotáxica y fagocítica de los neutrófilos monocitos y macrófagos, que a su vez generan complicaciones en la capacidad de enfrentar los riesgos biológicos potenciales, los cuales enfrentan los animales durante su vida productiva, provocando un riesgo sobre la capacidad productiva de un lote [2].

Las células inactivadas de Propionibacterium acnes y el lipopolisacárido de Eschericha coli poseen propiedades inmunoterapéuticas. La primera se caracteriza por inducir la producción de interferón y estimular los linfocitos murinos y humanos, con beneficios en enfermedades neoplásicas. El lipopolisacárido de E. coli induce la diferenciación de linfocitos B a células plasmáticas, produciendo los anticuerpos y estimulando los linfocitos T. La utilidad de este compuesto radica en la activación de la respuesta inmune específica en las mucosas con la secreción de inmunoglobulina A y en la respuesta humoral y celular con proliferación de linfocitos B y producción de anticuerpos [3].

Otra de las características de los inmunomoduladores es la forma en la cual se encuentran los aminoácidos y vitaminas, que permiten mayor biodisponibilidad y, por tanto, son más asimilables. De esta manera, están considerados como bioestimulantes muy completos para los animales en sus fases de producción [4].

Para que cada organismo desarrolle sus funciones de manera óptima necesita un nivel adecuado de vitaminas y aminoácidos que sean biodisponibles. Esto contribuye con los procesos de síntesis de nuevas moléculas, logrando una mejor respuesta inmune para realizar la síntesis de proteínas en la fase aguda de la infección; da lugar a que se logren los procesos de división y diferenciación celular, permitiendo una mayor capacidad de respuesta frente a los agentes patógenos. Puede ocurrir en el individuo un desequilibrio a nivel nutricional que afecte la funcionalidad del sistema inmune, y por ende el mantenimiento de la homeostasis inmunológica, además de una comunicación bidireccional errada en los sistemas endocrino y nervioso. Entre las principales vitaminas se encuentran la $\mathrm{A}$, $\mathrm{B}_{15}, \mathrm{~B}_{1}, \mathrm{~B}_{2}, \mathrm{~B}_{6}, \mathrm{~B}_{12}, \mathrm{D}_{3}, \mathrm{~K}_{3}$, y los principales aminoácidos son: ácido aspártico, fenilalamina, cistina, ácido glutámico, glicina, histidina, isoleucina, leucina, lisina, metionina, prolina, serina, treonina, triptofano, tirosina, valina [5].

La incidencia de la enfermedad de Gumboro tiene un carácter global, ya que se ha detectado en todas las zonas de producción intensiva de aves. Dicha incidencia es elevada y, por lo general, los lotes resultan infectados a una edad temprana, ya sea por infección natural o por vacunación [6].

En el presente trabajo se evaluó el efecto del peso de los órganos de las aves tratadas y sin tratar como 
método de diagnóstico al alcance de todos los técnicos de campo, para conocer la condición inmunológica de la parvada.

Cada individuo posee órganos y diferentes tipos de células de acuerdo con sus funciones, en este caso el sistema inmunitario (sI), donde está el bazo, el timo y la bursa, que se encuentran encapsulados por un tejido conectivo y actúan frente a moléculas extrañas, las cuales reaccionan al ingresar en el cuerpo [7].

\section{Materiales y métodos}

El estudio se realizó en la vereda San Nicolás, ubicada en el área rural del municipio de Lebrija, departamento de Santander, Colombia. La población de estudio la constituyeron aves de la raza Isa Brown, alojadas en galpones diferentes, en las que se identificó el grupo tratado A, con inmunomodulador, vitaminas y aminoácidos, y grupo control B (J7) con hepatoprotector.

Para el desarrollo del trabajo se identificaron dos grupos así: el grupo A (prueba J8) con 55.062 aves, al cual se le adicionó el inmunomodulador, vitaminas y aminoácidos.

El grupo B (control J7) con 55.484 aves, al que se le administró el hepatoprotector.

En los dos grupos se tomaron muestras serológicas para la entidad de Gumboro a cinco y nueve semanas.

Semanalmente fueron elegidas al azar tres aves por cada grupo, las cuales se pesaban y se les extraía muestra para serología de la enfermedad de Gumboro (semanas 5 y 9). Los órganos linfoides fueron extraídos y pesados.

Los materiales incluyeron los resultados obtenidos y procesamiento de la información, por medio de las pruebas de Elisa, peso de órganos linfoides (bazo, timo y bolsa de Fabricio) y resultados de parámetros zootécnicos, con el fin de establecer su influencia en el desempeño de las aves en la fase de levante.

\section{Análisis estadístico}

Los resultados se presentan por medio de gráficas longitudinales para las variables peso de la polla, del timo, la bolsa y el bazo; adicionalmente, se realizó la prueba de t para probar la hipótesis de diferencia en los promedios entre los tratamientos, de las mismas variables, en una base semanal. Las variables zootécnicas consumo, peso de la polla, uniformidad y conversión de los lotes se presentan por medio de tablas de estadística descriptiva. Para probar la hipótesis de diferencia en los promedios entre los tratamientos, en todo el periodo de estudio, se utilizó un modelo de bloques completos al azar, con submuestreo, en las variables peso de la polla, del timo, la bolsa y el bazo, en las que el efecto de bloque lo da la semana. Para las variables zootécnicas, se prueba la hipótesis de diferencia en los promedios, entre los tratamientos, en todo el periodo de estudio, por medio de un modelo de bloques completos al azar, con una replicación por bloque, en la que el efecto del bloque lo da la semana.

\section{Resultados}

Inicialmente, se presentan los resultados por medio de gráficas longitudinales para las variables peso de la polla, del timo, la bolsa y el bazo; luego se presentan los resultados de la estadística inferencial para todas las variables.

Se observa que la evolución del peso promedio del bazo es similar entre los dos grupos de aves, aunque el grupo J8 tuvo un ligero mayor peso promedio hasta la semana 6 (figura 1 ).

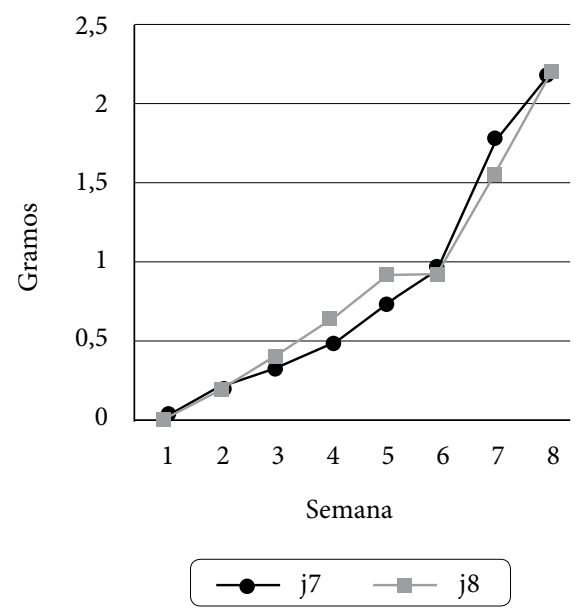

Figura 1. Evolución del peso promedio del bazo entre aves alimentadas con y $\sin$ inmunomodulador. En cada grupo $n=3$ Fuente: elaboración propia

En la figura 2 se observa una similitud en el peso del timo de los dos grupos, posterior a la cuarta semana, el grupo control hace un descenso en peso del timo y luego continúa un crecimiento mayor al del grupo prueba. 


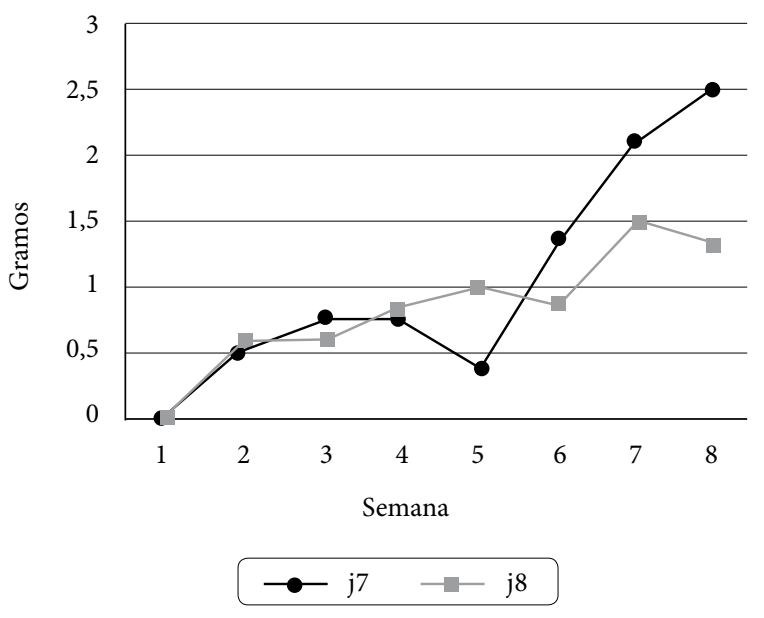

Figura 2. Evolución del peso promedio del timo en aves con y sin inmunomodulador

Fuente: elaboración propia

Se observó que el grupo prueba tiene un crecimiento continuo durante las primeras tres semanas, posterior a esta realiza descenso hasta seis semanas, para luego volver a crecer a siete semanas; en el grupo control el crecimiento presenta a dos semanas y entra en un periodo de mantenimiento del peso de la bolsa hasta seis semanas y en adelante crece hasta ocho semanas (figura 3).

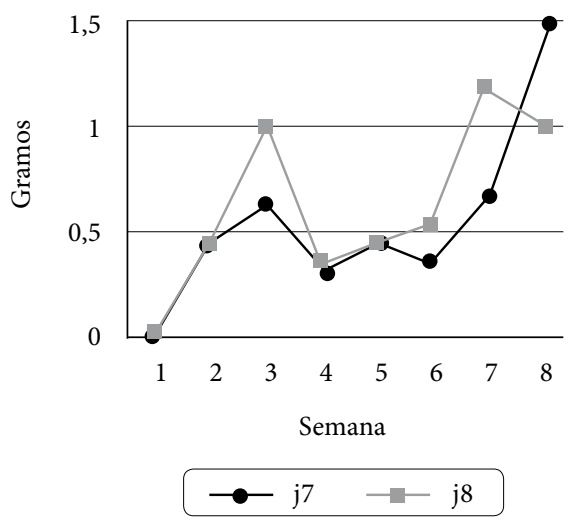

Figura 3. Evolución del peso de la bolsa de Fabricio entre los dos grupos de aves con y sin inmunomodulador Fuente: elaboración propia

Se observó en relación con el peso de las aves que el grupo prueba presentó un peso promedio mayor al grupo control, y al finalizar la octava semana se mantuvieron similares (figura 4).

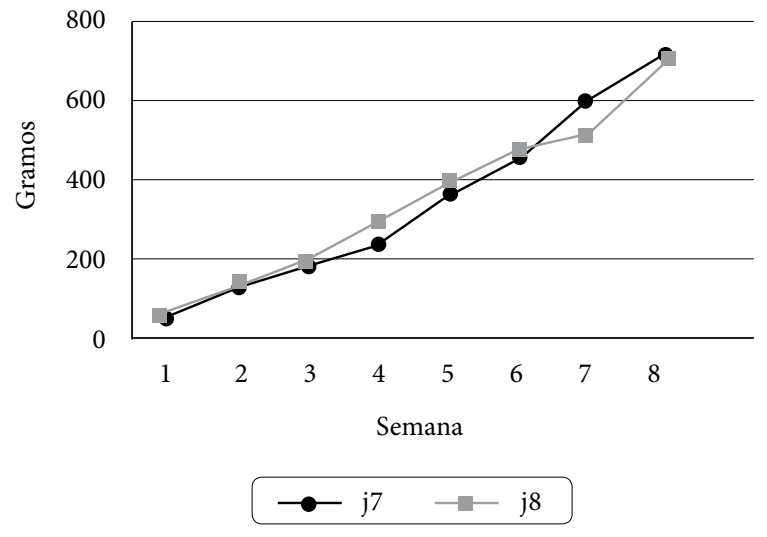

Figura 4. Evolución del peso promedio de las aves con y sin inmunomoduladores

Fuente: elaboración propia

Con respecto a los resultados de la prueba de hipótesis de t, para comparar las diferencias entre los promedios de las variables peso de la polla y peso del timo (tabla 1), se reportan diferencias significativas sólo para el peso de la polla únicamente en las semanas 1 y 4 $(\mathrm{P}<0,05)$.

Tabla 1. Promedios y desviación estándar para cada variable, en cada tratamiento, por semana. El promedio corresponde a tres aves en cada semana en cada tratamiento. Se incluyen los resultados de la prueba de hipótesis de t para la diferencia de promedios entre tratamientos, en cada semana, para cada variable

\begin{tabular}{|c|c|c|c|c|}
\hline \multirow{2}{*}{ 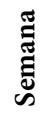 } & \multicolumn{2}{|c|}{ Peso } & \multicolumn{2}{|c|}{ Timo } \\
\hline & J7 & J8 & J7 & $\mathrm{J8}$ \\
\hline 1 & $63,7 \pm 0,667^{a}$ & $75 \pm 1,155^{\mathrm{b}}$ & $0,025 \pm 0,003^{a}$ & $0,022 \pm 0,002^{\mathrm{a}}$ \\
\hline 2 & $133,7 \pm 3,667^{a}$ & $131 \mathrm{a} \pm 2,082^{\mathrm{a}}$ & $0,547 \pm 0,058^{a}$ & $0,583 \pm 0,082^{\mathrm{a}}$ \\
\hline 3 & $183,3 \pm 26,667^{a}$ & $207 \pm 16,258^{a}$ & $0,776 \pm 0,225^{a}$ & $0,655 \pm 0,133^{\mathrm{a}}$ \\
\hline 4 & $246,7 \pm 8,819^{a}$ & $298,3 \pm 4,41^{b}$ & $0,774 \pm 0,199^{a}$ & $0,877 \pm 0,23^{\mathrm{a}}$ \\
\hline 5 & $356,7 \pm 24,037^{a}$ & $386,7 \pm 29,627^{a}$ & $0,415 \pm 0,131^{\mathrm{a}}$ & $1,003 \pm 0,138^{\mathrm{b}}$ \\
\hline 6 & $453,3 \pm 36,667^{a}$ & $480 \pm 11,547^{a}$ & $1,356 \pm 0,331^{\mathrm{a}}$ & $0,887 \pm 0,096^{\mathrm{a}}$ \\
\hline 7 & $586,7 \pm 37,565^{a}$ & $526,7 \pm 3,333^{a}$ & $2,08 \pm 0,451^{\mathrm{a}}$ & $1,548 \pm 0,191^{\mathrm{a}}$ \\
\hline 8 & $720 \pm 11,547^{a}$ & $710 \pm 57,735^{a}$ & $2,505 \pm 0,325^{a}$ & $1,388 \pm 0,515^{\mathrm{a}}$ \\
\hline \multicolumn{5}{|c|}{$\begin{array}{l}\text { Se presenta el promedio } \pm \text { error estándar de tres aves por grupo } \\
\text { Promedios con diferente letra indican diferencias significativas } \\
(\mathrm{P}<0,05) \text { entre los tratamientos en cada semana, para cada variable } \\
\text { en la prueba de } t\end{array}$} \\
\hline
\end{tabular}

Fuente: elaboración propia 
En relación con los resultados de la prueba de hipótesis de t, para comparar las diferencias entre los promedios de las variables peso de la polla y peso del timo (tabla 2), se reportan diferencias significativas sólo para el peso de la polla en las semanas 1 y 4 , y para el peso del timo en la semana $5(\mathrm{P}<0,05)$, pero no se reportan diferencias significativas en las variables peso de la bolsa de Fabricio y peso del bazo (tabla 3 ).

Tabla 2. Promedios y desviación estándar para cada variable, en cada tratamiento, por semana. El promedio corresponde a tres aves en cada semana en cada tratamiento. Se incluyen los resultados de la prueba de hipótesis de t para la diferencia de promedios entre tratamientos, en cada semana, para cada variable

\begin{tabular}{|c|c|c|c|c|}
\hline \multirow{2}{*}{ 径 } & \multicolumn{2}{|c|}{ Bazo } & \multicolumn{2}{|c|}{ Bolsa } \\
\hline & j7 & j8 & j7 & j8 \\
\hline 1 & $0,023 \pm 0,003^{a}$ & $0,026 \pm 0,002^{\mathrm{a}}$ & $0,04 \pm 0,003^{\mathrm{a}}$ & $0,03 \pm 0,003^{a}$ \\
\hline 2 & $0,188 \pm 0,022^{\mathrm{a}}$ & $0,197 \pm 0,026^{\mathrm{a}}$ & $0,45 \pm 0,114^{\mathrm{a}}$ & $0,44 \pm 0,143^{\mathrm{a}}$ \\
\hline 3 & $0,335 \pm 0,034^{\mathrm{a}}$ & $0,426 \pm 0,098^{a}$ & $0,63 \pm 0,231^{\mathrm{a}}$ & $1 \pm 0,141^{\mathrm{a}}$ \\
\hline 4 & $0,499 \pm 0.053^{a}$ & $0,673 \pm 0,104^{a}$ & $0,32 \pm 0,182^{a}$ & $0,34 \pm 0,057^{\mathrm{a}}$ \\
\hline 5 & $0,736 \pm 0,2^{\mathrm{a}}$ & $0,925 \pm 0,084^{\mathrm{a}}$ & $0,42 \pm 0,081^{\mathrm{a}}$ & $0,46 \pm 0,07^{a}$ \\
\hline 6 & $0,954 \pm 0,067^{a}$ & $0,932 \pm 0,109^{a}$ & $0,35 \pm 0,085^{a}$ & $0,53 \pm 0,037^{\mathrm{a}}$ \\
\hline 7 & $1,756 \pm 0,117 \mathrm{a}$ & $1,616 \pm 0,076^{a}$ & $0,67 \pm 0,154^{\mathrm{a}}$ & $1,18 \pm 0,267 \mathrm{a}$ \\
\hline 8 & $2,204 \pm 0,4^{\mathrm{a}}$ & $2,131 \pm 0,234^{\mathrm{a}}$ & $1,43 \pm 0,217^{\mathrm{a}}$ & $1 \pm 0,132^{\mathrm{a}}$ \\
\hline \multicolumn{5}{|c|}{$\begin{array}{l}\text { Se presenta el promedio } \pm \text { error estándar de tres aves por grupo } \\
\text { Promedios con diferente letra indican diferencias significativas } \\
(\mathrm{P}<0,05) \text { entre los tratamientos en cada semana, para cada variable } \\
\text { en la prueba de } \mathrm{t}\end{array}$} \\
\hline
\end{tabular}

Fuente: elaboración propia

La evolución semanal de las variables zootécnicas se presenta en la tabla 3. Los lotes no presentan grandes diferencias para las variables peso, conversión, uniformidad y consumo diario. El peso promedio de la polla fue ligeramente mayor en las semanas 4, 5, 7 y 8 , para el tratamiento J8.

Con respecto a los resultados del análisis de varianza (tabla 4), para el modelo de bloques completos al azar con submuestreos, para establecer diferencias en las variables peso de la bolsa, de la polla, del timo y del bazo, considerando la semana como efecto bloque, se halló que no hubo diferencias significativas en los promedios entre los tratamientos $(\mathrm{P}>0,05)$.
Tabla 3. Variables zootécnicas semanales, consumo diario, peso, uniformidad y conversión

\begin{tabular}{|c|c|c|c|c|c|c|c|c|}
\hline \multirow{2}{*}{ 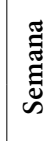 } & \multicolumn{2}{|c|}{$\begin{array}{l}\text { Consumo } \\
\text { diario(g) }\end{array}$} & \multicolumn{2}{|c|}{ Peso (g) } & \multicolumn{2}{|c|}{ Uniformidad } & \multicolumn{2}{|c|}{ Conversión } \\
\hline & $\mathrm{J} 7$ & J8 & J7 & J8 & $\mathrm{J} 7$ & $\mathrm{~J} 8$ & J7 & J8 \\
\hline 1 & 10,7 & 11,73 & 68,4 & 75 & 74 & 67 & 0,16 & 0,16 \\
\hline 2 & 15,5 & 14,97 & 132,2 & 132,5 & 68 & 65 & 0,12 & 0,11 \\
\hline 3 & 20,9 & 21,95 & 200 & 207 & 80,2 & 80 & 0,10 & 0,11 \\
\hline 4 & 28,68 & 27,68 & 294,7 & 300,6 & 60,7 & 80,7 & 0,10 & 0,09 \\
\hline 5 & 33,35 & 34,25 & 377 & 393,1 & 76,4 & 77 & 0,09 & 0,09 \\
\hline 6 & 39,46 & 39,48 & 517,5 & 497,3 & 68 & 78 & 0,08 & 0,08 \\
\hline 7 & 47,95 & 47,21 & 620,4 & 623,5 & 79,1 & 79,73 & 0,08 & 0,08 \\
\hline 8 & 47,98 & 49,75 & 693,7 & 724,7 & 80 & 77 & 0,07 & 0,07 \\
\hline
\end{tabular}

Fuente: elaboración propia

Tabla 4. Resultados del análisis de varianza para el modelo de bloques completos al azar con submuestreo, para las diferencias entre los tratamientos, en los promedios del peso de la bolsa, peso del ave, peso del timo y peso del bazo, considerando el efecto de la semana como bloque. El número de submuestras fue de 3 en cada tratamiento

\begin{tabular}{|l|c|c|c|c|}
\hline Fuente & G.l. Núm. & G.l. Den. & F & Valor P \\
\hline Bolsa & 1 & 7 & 0,543 & 0,485 \\
\hline Peso & 1 & 7 & 0,740 & 0,418 \\
\hline Timo & 1 & 7 & 1,091 & 0,331 \\
\hline Bazo & 1 & 7 & 0,013 & 0,877 \\
\hline
\end{tabular}

Fuente: elaboración propia

Con respecto a los resultados del análisis de varianza (tabla 5), para el modelo de bloques completos al azar con una sola replicación, para establecer diferencias en las variables zootécnicas, consumo, peso de la polla, uniformidad y conversión, considerando la semana como efecto bloque, se halló que no hubo diferencias significativas en los promedios entre los tratamientos $(\mathrm{P}>0,05)$.

Tabla 5. Resultados del análisis de varianza para el modelo de bloques completos al azar con una sola replicación, para las diferencias entre los tratamientos, en los promedios de las variables zootécnicas, consumo diario, peso de la polla, uniformidad y conversión. Los datos corresponden al resumen de todo el lote

\begin{tabular}{|l|l|l|l|l|}
\hline \multicolumn{1}{|c|}{ Fuente } & G.l. Núm. & G.l. Den. & \multicolumn{1}{c|}{ F } & \multicolumn{1}{c|}{ Valor P } \\
\hline Consumo & 1 & 7 & 0,765 & 0,411 \\
\hline Peso polla & 1 & 7 & 1,49 & 0,262 \\
\hline Uniformidad & 1 & 7 & 0,540 & 0,487 \\
\hline Conversión & 1 & 7 & 1,116 & 0,326 \\
\hline
\end{tabular}

Fuente: elaboración propia 


\section{Serologías}

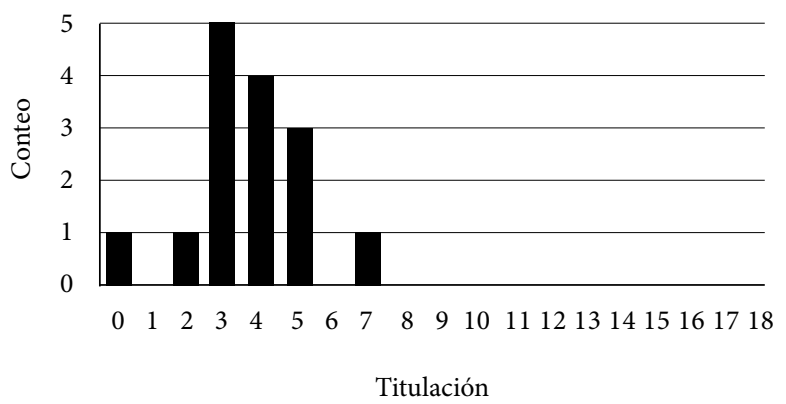

Figura 5. Serología 5 SEM J8 (Grupo Prueba) Gumboro (IBD) Fuente: elaboración propia

\section{Conteo: 15}

Promedio: 3.424

Desviación estándar: 1.648

\%CV: 48,1

Mín: 1

Máx: 7.910

Dil: 1:500

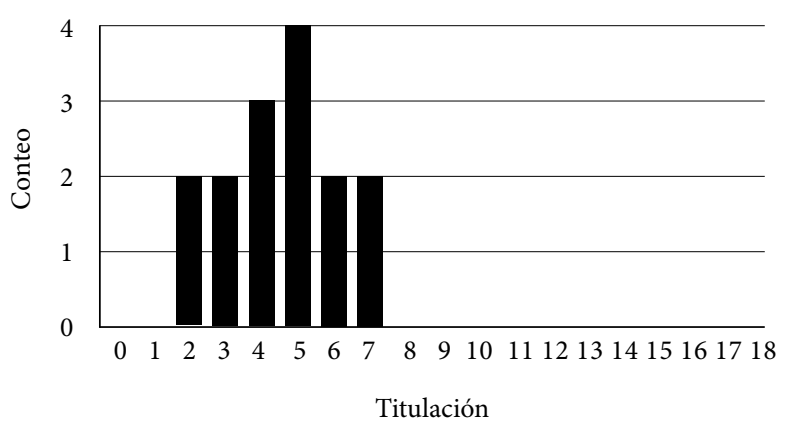

Figura 6. Serología 9 sem J8 (Grupo Prueba) Gumboro (IBD) Fuente: elaboración propia

\section{Conteo: 15}

Promedio: 3.980

Desviación estándar: 1.419

\%CV: 35,6

Mín: 1.592

Máx: 6.597

Dil: 1:500

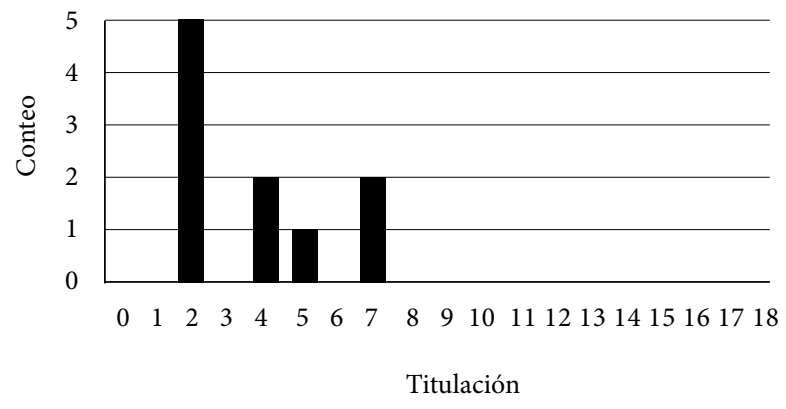

Figura 7. Serología 5 SEM J7 (Grupo Control) Gumboro (IBD) Fuente: elaboración propia

Conteo: 10

Promedio: 3.323

Desviación estándar: 2.046

\%CV: 61,6

Mín: 1.408

Máx: 7.010

Dil: 1:500

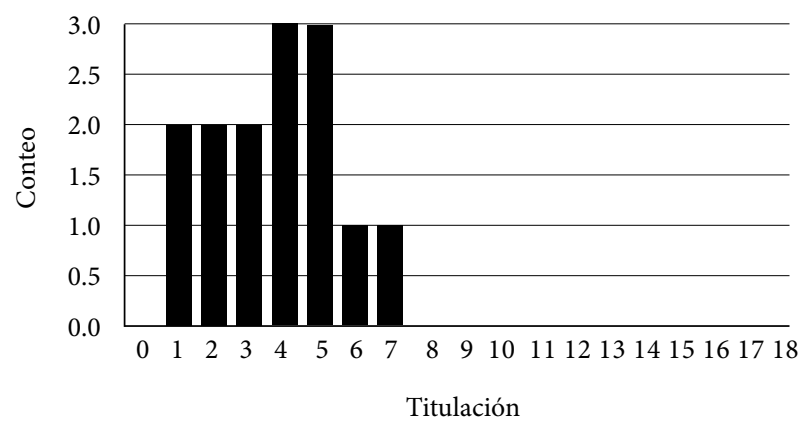

Figura 8. Serología 9 SEM J7 (Grupo Control) Gumboro (IBD) Fuente: elaboración propia

\section{Conteo: 14}

Promedio: 3.182

Desviación estándar: 1.784

\%CV: 56,0

Mín: 475

Máx: 6.988

Dil: 1:500 
Respecto a los títulos, tanto para J8 como para J7, se encuentran dentro de un buen perfil, indicando un método de vacunación adecuado, con una dosis apropiada de vacuna y planes de bioseguridad.

El Coeficiente de Variación (cv), tanto en la serología de la semana 5 como en la serología de la semana 9, es menor en el grupo J8 (tratados), lo que nos indica menos dispersión de los títulos que es resultado de un adecuado control vacunal para Gumboro y mayor uniformidad y homogeneización, lo que se traduce en mayor protección antigénica.

El CV del grupo J7, especialmente a la semana 5, presenta una elevada dispersión con títulos bastante heterogéneos, disminuyendo la protección antigénica para Gumboro.

Comparando los títulos de la semana 9, se observa un mejor perfil en las aves J8 (prueba). Este fenómeno está directamente relacionado con el bajo cV de este mismo grupo de aves.

\section{Discusión}

El comportamiento de los parámetros zootécnicos con respecto al consumo, conversión y uniformidad no presentaron grandes diferencias, y el peso promedio de las aves tuvo fluctuaciones en las semanas 4, 5, 7 y 8 en el grupo J8 (prueba).

El aumento del tamaño de estos órganos, especialmente en la bursa, donde se presentan pliegues que contienen numerosos folículos linfoides en los que se concentran y ordenan en mayoría células linfocitarias en dos zonas, medular y cortical, incrementa la población de células que participan en la respuesta inmunitaria, en especial células B. El mismo proceso ocurre en el timo, en el que se genera la diferenciación de linfocitos $\mathrm{T}$ ante la presencia de un antígeno, por el cual se permite una mejor respuesta inmune.

Esto fue demostrado por la prueba de Elisa posterior a la vacunación, para verificar el incremento en anticuerpos, que se presentó con una mejor respuesta en el grupo de la prueba.

En cuanto a los pesos de los órganos linfoides se presentó un mejor desarrollo y uniformidad de estos en el grupo prueba J8, lo que confirma la efectividad del inmunomodulador y su acción en el sistema linfoide. En relación con el grupo control J7, se presentaron pesos muy bajos al inicio, lo que permite concluir que necesita de un estimulante para tener mejor desarrollo $y$, por ende, una mejor respuesta inmunitaria frente a un desafío de campo.

Se considera que la respuesta inmune que genera el uso del inmunomodulador está dada por varios procesos fisiológicos que dan lugar a una elevada activación y reclutamiento de las células inmunitarias, particularmente las células fagocíticas; además, ayuda a acelerar el desarrollo y maduración del sistema inmune, incrementando la diversificación del repertorio de linfocitos y disminuyendo las consecuencias catabólicas de las infecciones que causan inmunosupresión.

Los inmunomoduladores tienen acciones específicas e inespecíficas porque ayudan a la activación de macrófagos (producción de citosinas que desencadenan la cascada de la inmunidad, aprovechando el efecto benéfico de este sistema de comunicación intercelular), células $\mathrm{T}$ y la producción de interluquina 2(IL-2), y, adicionalmente, estimulan la maduración de células T у B [7].

Los resultados obtenidos en las muestras serológicas indican que el grupo J8 presentó mayor respuesta uniforme a la inmunización contra Gumboro. La vacunación contra Gumboro "prendió" mucho mejor en el grupo de aves 58 .

Los lotes no presentan grandes diferencias para las variables conversión, uniformidad y consumo diario. El peso promedio de la polla fue ligeramente mayor en las semanas 4, 5, 7 y 8, para el tratamiento J8.

\section{Referencias}

[1] Nilipour A. Cómo eliminar Gumboro, una experiencia de campo. En: Hipra, editor. El libro blanco de la enfermedad de Gumboro. Girona (España): Gabinet Disseny Grafic; 2006. p. 75.

[2] Perozo F, Nava J, Mavárez Y, Arenas E, Serje P, Briceño M. Caracterización morfométrica de los órganos linfoides en pollos de engorde de la línea Ross criados bajo condiciones de campo en el estado de Zulia, Venezuela. Rev Cient (Maracaibo). 2004; 14(3): 217-25.

[3] Zekaria D, Romero R, Parada M, Silvestrim A, Rodríguez-Arrioja GM. Estudio del efecto de un inmunoterápico sobre la productividad de pollos de engorde y ponedoras. s. d. [Consultado: 29 de mayo del 2013]. Disponible en: http://www.calier.es/pdf/Inmunair\%20 Zekaria\%20et\%20al.pdf

[4] Soy del campo. Promocalier L. [Consultado: $12 \mathrm{de}$ abril del 2013]. Disponible en: http://www.soydel 
campo.com/vademecum_veterinario/productos. php?id=3031\&prod=PROMOCALIER-L

[5] Nova E, Montero A, Gómez S, Marcos A. La estrecha relación entre la nutrición y el sistema inmunitario. s. d. [Consultado: 22 de marzo del 2013]. Disponible en: http://www.seom.org/seomcms/images/stories/recursos/infopublico/publicaciones/soporteNutricional/ pdf/cap_01.pdf
[6] Banda A. Situación global del IBD. En: Hipra, editor. El libro blanco de la enfermedad de Gumboro. Girona (España): Gabinet Disseny Grafic; 2006. p. 15.

[7] Alzola R. Tejido hemocitopoyetico linfoide. 2002 [Consultado: 12 de febrero del 2013]. Disponible en: http://www.vet.unicen.edu.ar/html/Areas/HistEmbrTera/2009/Tejido.pdf 